\title{
The Top Quark
}

\author{
L. Di Lella
}

CERN, Geneva, Switzerland

\begin{abstract}
The Standard Model requires the existence of a sixth or top quark to suppress transitions of the fifth or beauty quark found in 1974. Prospects for its discovery are considered in the light of recent results showing that the top's mass exceeds $89 \mathrm{GeV} / \mathrm{c}^{2}$.
\end{abstract}

The quark model was proposed in 1964 [1] to describe the large number of strongly interacting particles (hadrons) produced at high-energy accelerators in terms of a small number of elementary constituents. In its original formulation, the model needed three quarks of spin $1 / 2$ and baryon number 1/3: the u-quark, with electric charge $+2 / 3$ (in units of the proton charge), and two quarks with electric charge $-1 / 3$, named $d$ and $s$. These quarks together with their antiquarks $\overline{\mathrm{u}}, \overline{\mathrm{d}}$ and $\overline{\mathrm{s}}$ were sufficient to describe all known baryons and mesons, the baryons consisting of three quarks and the mesons of a quarkantiquark pair. The $s$ and $\overline{\mathrm{s}}$ quarks were needed to account for the strange hadrons, such as the K-mesons and the hyperons $(\Lambda, \Sigma, \Xi, \Omega$, etc.).

In the quark model, the weak decay of a hadron is described in terms of the weak decay of one of the constituent quarks. For example, the $\beta$-decay of the neutron, which is a ddu system, is the result of the decay $d \rightarrow u e^{-} \bar{v}_{e}$ of one of the two d-quarks. The final quark system, duu, is the proton. Similarly, the $\beta$-decay of the $\Lambda$ hyperon (a uds system) is described in terms of quark decay s $\rightarrow$ $u^{-} \bar{v}_{e}$, resulting in the observed decay $\Lambda \rightarrow \mathrm{pe}^{-} \overline{\mathrm{v}}_{\mathrm{e}}$.

\section{Fourth and Fifth Quarks}

The existence of weak $s \rightarrow u$ transitions implied the occurrence of $s \rightarrow d$ transitions to second order in the weak interaction, as a result of an intermediate transition to a u-quark state ( $\mathrm{s} \rightarrow$ $\mathrm{u}, \mathrm{u} \rightarrow \mathrm{d})$. Such $\mathrm{s} \rightarrow \mathrm{d}$ transitions would result, for example, in the decays $\mathrm{K}_{\mathrm{L}}^{\circ} \rightarrow$ $\mu^{+} \mu^{-}$or $\mathrm{K}^{+}-\pi^{+} \mathrm{e}^{+} \mathrm{e}^{-}$which had not been observed experimentally in the 60 's (such decays were later measured to occur at very low rates, representing

Luigi Di Lella, an experimental high energy particle physicist, is the spokesperson for the UA2 Collaboration at CERN, $\mathrm{CH}-1211$ Geneva 23, Switzerland. He was educated at the Università di Pisa and at the Scuola Normale Superiore, Pisa, and joined the CERN staff in 1971 after holding temporary posts in Rome and at CERN and Columbia University, USA. $\approx 2.7 \times 10^{-7}$ and $9.5 \times 10^{-9}$ of the total $\mathrm{K}_{\mathrm{L}}^{0}$ and $\mathrm{K}^{+}$decay rates, respectively).

This problem was solved by Glashow, Iliopoulos and Maiani [2] who postulated the existence of a fourth quark of charge $+2 / 3$, which they called $c$ (for "charm"). In their model, often referred to as the GIM mechanism, the amplitude of the second-order transition $\mathrm{s} \rightarrow$ $\mathrm{u}, \mathrm{u} \rightarrow \mathrm{d}$ was almost completely cancelled by the amplitude of the transition $s \rightarrow c, c \rightarrow d$ (the cancellation would have been exact in the limit of equal $\mathrm{u}$ and $\mathrm{c}$ masses). On the basis of the first measurement of the $\mathrm{K}_{\llcorner}^{\circ} \rightarrow \mu^{+} \mu^{-}$ decay rate in the early 70's, it was possible to predict a value between 1 and $2 \mathrm{GeV} / \mathrm{c}^{2}$ for the mass of the c-quark.

The GIM mechanism received a beautiful experimental confirmation in November 1974 when two independent experiments, one performed at the Alternating Gradient Synchrotron of Brookhaven National Laboratory and the other at the Stanford $\mathrm{e}^{+} \mathrm{e}^{-}$collider SPEAR, discovered a new neutral meson with a mass of $\approx 3.1 \mathrm{GeV} / c^{2}$. The properties of this meson, called $\mathrm{J}$ at Brookhaven and $\Psi$ at Stanford, were such that the only plausible interpretation was in terms of a bound $c \bar{c}$ state. Shortly afterwards, other mesons containing c-quarks were observed at SPEAR [3].

The discovery of the c-quark made the number of quarks equal to the number of leptons which were known at that time $\left(e^{-}, v_{e^{\prime}} \mu^{-}, v_{\mu}\right)$. However, this apparent symmetry was soon broken by the discovery at SPEAR of a new heavy lepton, called $\tau^{-}$, and of its antiparticle $\tau^{+}$with a mass of $\sim 1.78 \mathrm{GeV} / c^{2}$. The $\tau$-lepton was always found to decay into final states containing a neutrino, de- monstrating that it has a quantum number different from that of the muon or the electron (otherwise decays such as $\tau^{-} \rightarrow \mathrm{e}^{-} \gamma$ or $\mu^{-} \gamma$ would be possible). Thus, the study of the $\tau$ decay properties revealed at the same time the existence of a third type of neutrino, $v_{\text {. }}$.

Evidence for a fifth quark was obtained in 1977 at the Fermilab proton synchrotron [4] by the discovery a new neutral meson $\mathrm{Y}$ with a mass of 9.46 $\mathrm{GeV} / \mathrm{c}^{2}$ and properties as expected for a bound state of a new quark-antiquark pair. Further experiments at $\mathrm{e}^{+} \mathrm{e}^{-}$colliders have demonstrated that this new quark, called b-quark, has charge $-1 / 3$; additional mesons containing b-quarks have since been observed [5].

\section{The Standard Model}

Our current picture of matter at the most elementary level is of fundamental fermions (particles of spin 1/2) interacting via exchange of spin 1 bosons. While $\gamma$-exchange is responsible for the electromagnetic interaction, three heavy bosons $\mathrm{W}^{+}, \mathrm{W}^{-}$and $\mathrm{Z}$ are needed to describe all known weak processes. Each quark type, or "flavour", exists in three states which differ by a property named "colour". Quarks interact strongly by the exchange of massless vector bosons (the gluons), which exist in eight colour states. The quantum field theory of quarks and gluons, called Quantum Chromodynamics or $\mathrm{QCD}$, represents the modern theory of the strong interaction.

This picture is known as the Standard Model of the elementary particles. In the case of a weak interaction mediated by the exchange of $\mathrm{W}$ bosons, it requires that the fermions be arranged in doublets, as shown in Table 1, with

Table 1 - The fundamental particles (spin 1/2) and their approximate masses (in $\mathrm{MeV} / \mathrm{c}^{2}$ ).

\begin{tabular}{|c|c|c|c|c|c|c|c|c|c|c|}
\hline & \multicolumn{3}{|c|}{ 1st family } & \multicolumn{3}{|c|}{ 2rd family } & \multicolumn{3}{|c|}{ 3rd family } & Charge \\
\hline \multirow[t]{3}{*}{ Leptons } & \multicolumn{3}{|c|}{ electron neutrino } & \multicolumn{3}{|c|}{ muon neutrino } & \multicolumn{3}{|c|}{ tau neutrino } & \\
\hline & & $v_{e}$ & $<10 \mathrm{eV}$ & & $v_{\mu}$ & $<0.3$ & & & $<30$ & 0 \\
\hline & electron & $e^{-}$ & 0.51 & muon & $\mu^{-}$ & 106 & tau & & 1784 & -1 \\
\hline \multirow[t]{2}{*}{ Quarks } & up & $\mathrm{u}$ & 5 & charm & c & 1350 & top & & 89000 & $2 / 3$ \\
\hline & down & d & 10 & strange & s & 200 & bottom & & 4500 & $-1 / 3$ \\
\hline
\end{tabular}


weak transitions occurring between upper and lower members (these doublets are often referred to as "weak isospin doublets"). This structure implies the existence of a sixth quark (the top quark). Such a quark is needed to suppress $b \rightarrow s$ and $b \rightarrow d$ transitions, just as the c-quark was needed to suppress the $s \rightarrow d$ transition. Furthermore, the top quark ensures that the sum of all fermion electric charges be zero (when performing this sum, each quark charge must be multiplied by 3 to take into account the three colour states). It has been demonstrated [6] that this condition is necessary for the weak interaction theory to be renormalizable (the property of renormalization allows the calculation of transition amplitudes to any order in terms of a finite number of measurable parameters). However, experimental searches at $\mathrm{e}^{+} \mathrm{e}^{-}$and $\overline{\mathrm{p}} \mathrm{p}$ colliders have failed to find any evidence for the production and decay of the top quark. The most recent experiments, reviewed in the next two sections, have established significant lower bounds for the mass value of the top quark, $m_{\text {top }}$.

\section{Searches at $\mathbf{e}^{+} \mathbf{e}^{-}$Colliders}

Quarks have never been detected as free particles because of the long-distance behaviour of the strong force among coloured particles, which confines the quarks inside the observed, colourless hadrons (the ordinary mesons and baryons).

At $\mathrm{e}^{+} \mathrm{e}^{-}$colliders the process $\mathrm{e}^{+} \mathrm{e}^{-}$ $\rightarrow$ hadrons can be described as a twostep process. The first step is the elementary reaction

$$
\mathrm{e}^{+} \mathrm{e}^{-} \rightarrow \overline{\mathrm{q}} \mathrm{q}
$$

where $\mathrm{q}$ and $\overline{\mathrm{q}}$ are a quark and an antiquark of the same flavour. The crosssection for this reaction, which involves the exchange of a virtual $\gamma$ or $Z$ boson (see Fig. 1a), can be calculated as a function of the total centre-of-mass energy $\sqrt{ } s \equiv 2 E_{\text {beam }}$ and of the quark mass, $m_{q}$ (energy-momentum conservation requires $\sqrt{ } s>2 m_{\mathrm{q}} c^{2}$ ).

The second step involves the strong interaction between the outgoing $q$ and $\bar{q}$, responsible for colour confinement, which results in the production of many hadrons (this step is referred to as hadronization, or fragmentation). Since this is a long-distance interaction, it involves only small momentum transfers. As a consequence, the outgoing hadrons are produced mostly along the directions of the outgoing $q$ and $\bar{q}$, resulting in two rather collimated systems of hadrons (jets). Jet production is the dominant feature of hadronic final states from high-energy $\mathrm{e}^{+} \mathrm{e}^{-}$collisions (see cover illustration). a)
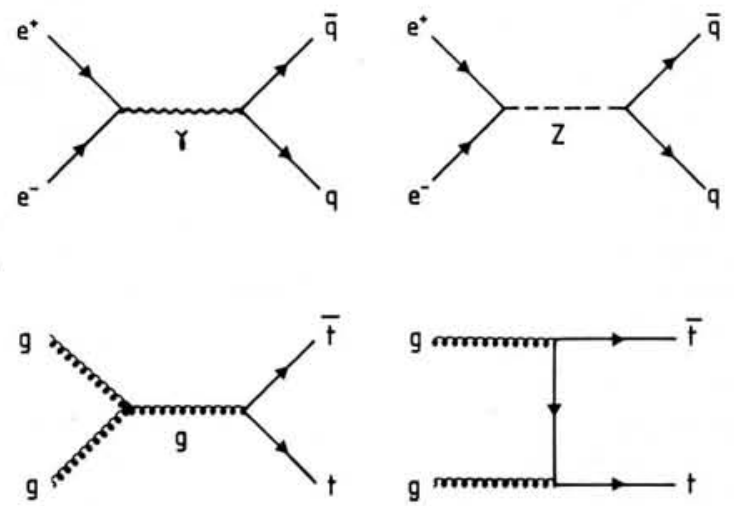

b)

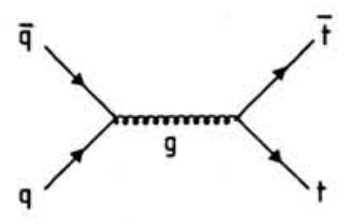

c)
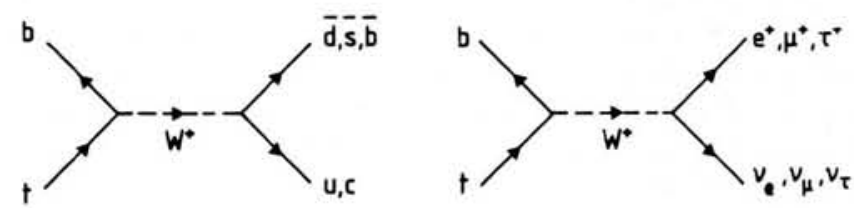

Fig. 1 - Leading-order $Q C D$ diagrams for: a) the production of a quark-antiquark pair from $e^{+} e^{-}$collisions; $b$ ) the production of a t $\bar{t}$ pair from $\bar{p} p$ collisions (and, more generally, from hadronic collisions); c) for hadronic and leptonic decays of a top quark.

Fig. 2 - The cross-section for hadron production from $\mathrm{e}^{+} \mathrm{e}^{-}$collisions, normalized to $\mu^{+} \mu^{-}$production, at three different colliders as a function of the total centre-of-mass energy $\sqrt{ } s$. The full curve (dashed curve) is the theoretical prediction excluding (including) the production of $t \bar{t}$ pairs. The data agree with an analysis based upon the production of only the first five quark flavours and excluding a top quark with a mass $<25.9$ $\mathrm{GeV} / \mathrm{c}^{2}$.

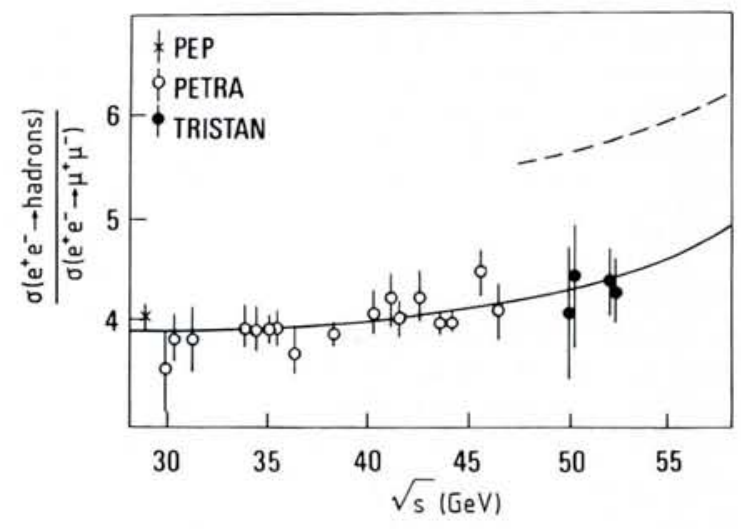

There are two ways to search for the top quark in $\mathrm{e}^{+} \mathrm{e}^{-}$collisions. The first consists in measuring the rate for the process $\mathrm{e}^{+} \mathrm{e}^{-} \rightarrow$ hadrons and comparing it with the expected rate, calculated with or without top production. An example of this approach is illustrated in Fig. 2, which shows the measured hadronic event rate from experiments at three different $\mathrm{e}^{+} \mathrm{e}^{-}$colliders [7] (this rate is normalized to the measured rate of $\mathrm{e}^{+} \mathrm{e}^{-} \rightarrow \mu^{+} \mu^{-}$events to reduce systematic uncertainties). The data points agree well with a prediction which assumes that only the first five quark flavours are produced, and disagree with a prediction that includes the top quark. The results of Fig. 2 alone exclude the existence of a top quark with a mass value lower than $25.9 \mathrm{GeV} / \mathrm{c}^{2}$.

The second method relies on the expected decay properties of a heavy top quark. In the Standard Model, top quark decays are due to the weak interaction (see the diagram of Fig. 1c). For top masses smaller than the $W$ mass $\left(m_{w}\right.$ $\left.\approx 80 \mathrm{GeV} / c^{2}\right)$, the decay rate is proportional to $m_{\text {top }}^{5}$, resulting in a very short 
mean life (of the order of $3 \times 10^{-20} \mathrm{~s}$ for $m_{\text {top }}=40 \mathrm{GeV} / c^{2}$ ), so only the decay secondaries can be detected. Because of the large top mass, such secondaries are emitted, in general, at large angles with respect to the original direction of the top quark, resulting in final-state configurations which do not show the two-jet structure typical of hadronic events associated with the production of light quarks.

Final-state configurations are studied by defining suitable event shape variables, calculated for each event from the measured momentum vectors of the outgoing secondaries, and take different values for two-jet events or for isotropic multi-particle final states. The production and decay of heavy top quarks would then significantly modify the distributions of such variables with respect to those expected if only the first five quark flavours are produced.

Similarly, in the leptonic decays of a heavy top quark $\left(\mathrm{t} \rightarrow\right.$ be $^{+} v_{\mathrm{e}}$ or $\mathrm{t} \rightarrow$ $b \mu^{+} v_{\mu}$ ) the charged leptons (e or $\mu$ ) are emitted, in general, at large angles with respect to the other hadrons. These isolated leptons are a typical feature of top quark decay, contrary to the leptonic decay of light quarks produced with energies $E \gg m_{c} c^{2}$.

Recently, the LEP experiments have searched for a top signal in the distributions of various event shape variables, and also by detecting isolated charged leptons [9]. The data agree with expectations from the five known quark flavours and show no evidence for a top signal. These results exclude the existence of a top quark with a mass value lower than $\approx 45 \mathrm{GeV} / c^{2}$.

\section{Searches at $\bar{p} \mathbf{p}$ Colliders}

Proton and antiproton beams can be described as wide-band beams of quarks, antiquarks and gluons. For a given $\mathrm{p}$ and $\overline{\mathrm{p}}$ momentum, the momentum distributions of these elementary constituents are reasonably well known from the results of deep-inelastic lepton-nucleon scattering experiments.

At a $\bar{p} p$ collider, the top quark can be produced from two dominant processes, mediated either by the weak interaction

$$
\begin{aligned}
& \overline{\mathrm{p}} \mathrm{p} \rightarrow \mathrm{W}^{ \pm}+\text {anything else; } \\
& \mathrm{W}^{+} \rightarrow \mathrm{tb} \text { or } \mathrm{W}^{-} \rightarrow \overline{\mathrm{tb}},
\end{aligned}
$$

or by the strong interaction

$$
\overline{\mathrm{p}} \mathrm{p} \rightarrow \overline{\mathrm{tt}}+\text { anything else. }
$$

The first process has a measurable rate if $\mathrm{W}^{+} \rightarrow$ tb is energetically allowed. In this case, its rate can be reliably calculated from the measured rate of $\mathrm{W} \rightarrow \mathrm{ev}_{\mathrm{e}}$ decays:

Fig. 3 - Predicted cross-sections for the production of a $t$ or $\bar{t}$ quark from $\bar{p} p$ collisions at total centre-of-mass energies of 0.63 and $1.8 \mathrm{TeV}$ corresponding to the CERN (upper) and Fermilab (lower) $\bar{p} p$ colliders, respectively. The bands represent the size of the theoretical uncertainty on the estimate of $t \bar{t}$ production from the strong interaction: the dashed curves represent the contributions from $t \bar{t}$ production alone.

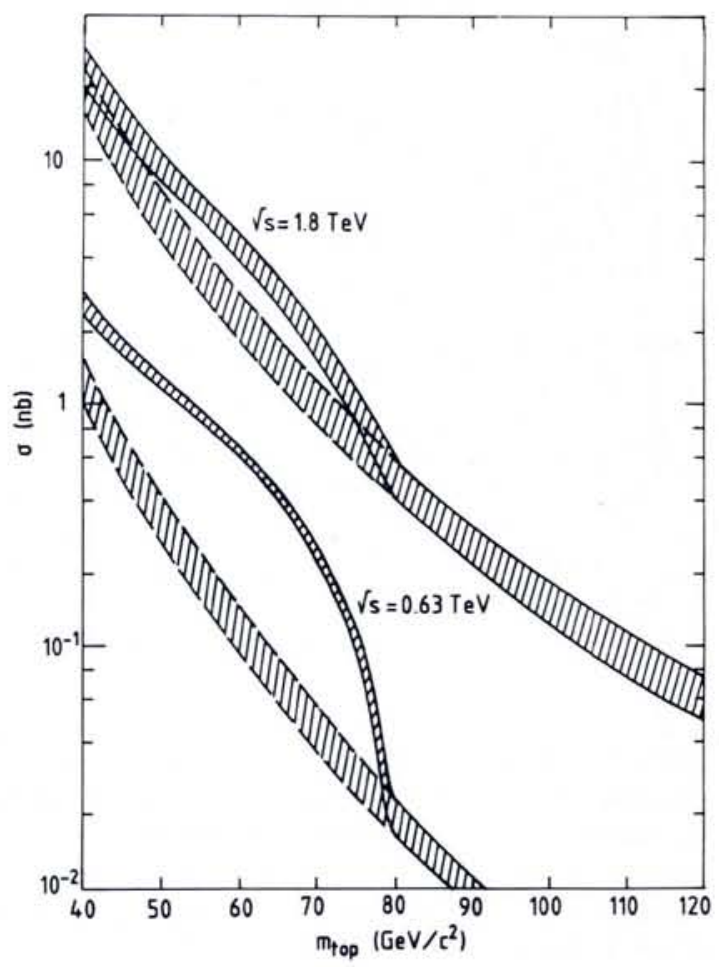

$\mathrm{R}\left(\mathrm{W}^{+} \rightarrow \mathrm{tb}\right)=$

$3 \mathrm{R}\left(\mathrm{W}^{+} \rightarrow \mathrm{e}^{+} v_{\mathrm{e}}\right) \phi\left(m_{\mathrm{top}}\right) C_{\mathrm{OCD}}$

where the factor 3 relates the production of coloured quarks to leptons, $\phi\left(m_{\text {top }}\right)$ is a phase-space factor which depends on $m_{\text {top }}$ and $C_{\mathrm{OCD}}$ is a QCD correction which is important only if $m_{\text {top }}$ is close to $m_{w}$.

Process (3) is a result of the elementary reactions of gluon-gluon fusion and $\mathrm{q} \bar{q}$ annihilation (see the diagrams of Fig. 1b). The cross-section for this process has been evaluated [10] using the full next-to-leading order QCD calculation. The theoretical uncertainties affecting the results of this calculation amount to approximately $\pm 30 \%$.

Fig. 3 shows the sum of the contributions from process (2) and (3) for $\sqrt{ } s=$ 0.63 and $1.8 \mathrm{TeV}$, the total centreof-mass energy values of the CERN and Fermilab $\bar{p} p$ colliders, respectively. A comparison between the two curves in Fig. 3 shows clearly the advantage of the higher-energy collider when searching for a heavy top quark. For equal machine luminosities, the production rate of top quarks at $\sqrt{ } s=1.8 \mathrm{TeV}$ exceeds that expected at $\sqrt{ } s=0.63$ $\mathrm{TeV}$ by at least one order of magnitude, and by much larger factors for the case $m_{\text {top }}>m_{w}$.

At $\bar{p} p$ colliders, the decay of a top quark into hadrons is very difficult to distinguish from the large background owing to strong interaction processes. Searches for top are therefore performed using the leptonic decay modes $t \rightarrow$ be $^{+} v_{e}$ or $t \rightarrow b \mu^{+} v_{\mu}$, each having a branching ratio of $1 / 9$ in the Standard Model. In the case of process (2), the signature of top production is a final state containing an isolated charged lepton (e or $\mu$ ) and two jets associated with the $b$ and $\bar{b}$ quarks. For process (3) the final state contains either a charged lepton and four jets (if the $\overline{\mathrm{t}}$ decays hadronically), or two charged leptons of opposite charge signs and two jets if both $t$ and $\bar{t}$ decay leptonically. Outgoing neutrinos escape detection and give rise, in general, to a measurable transverse momentum imbalance (missing $p_{\mathrm{T}}$ ), which can be used as an additional selection requirement for top events (the momentum imbalance along the beam axis cannot be measured because of the large number of undetected particles emitted at smal angles to the beam axis inside the machine vacuum pipe).

In addition to the particles resulting from top decay, the collisions of the other quarks, antiquarks and gluons contained in the incident $p$ and $\bar{p}$ resuit in a large number of hadrons. These hadrons, which are absent in $\mathrm{e}^{+} \mathrm{e}^{-} \mathrm{col}-$ lisions, have generally a rather low transverse momentum (of the order of $0.5 \mathrm{GeV} / c^{2}$ on average). They are often given the global name of "underlying event".

\section{Experiments}

Three experiments have recently searched for the top quark at $\bar{p} p$ colliders: the UA1 and UA2 experiments at CERN, and the Collider Detector at 


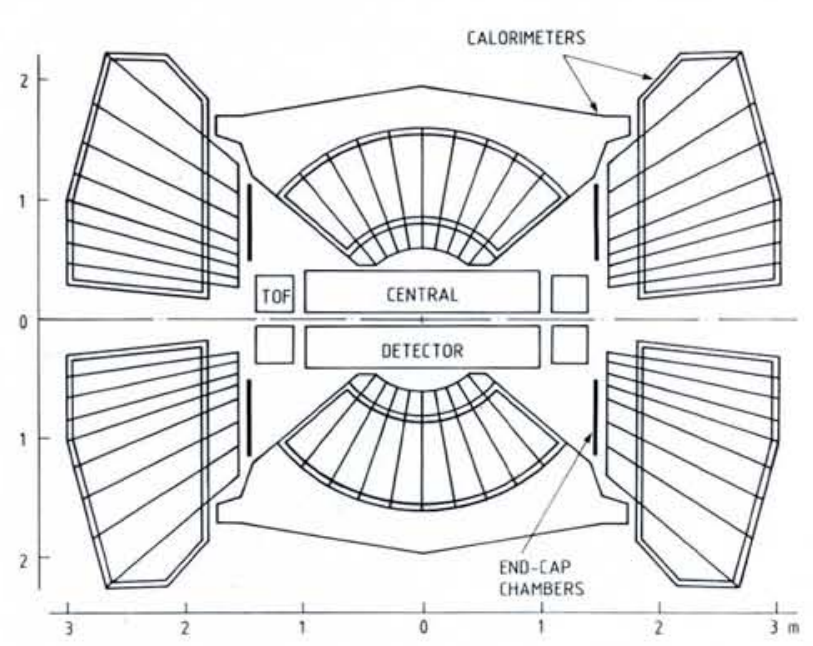

Fig. $4-A$ view of the UA2 detector in a plane containing the beam line.

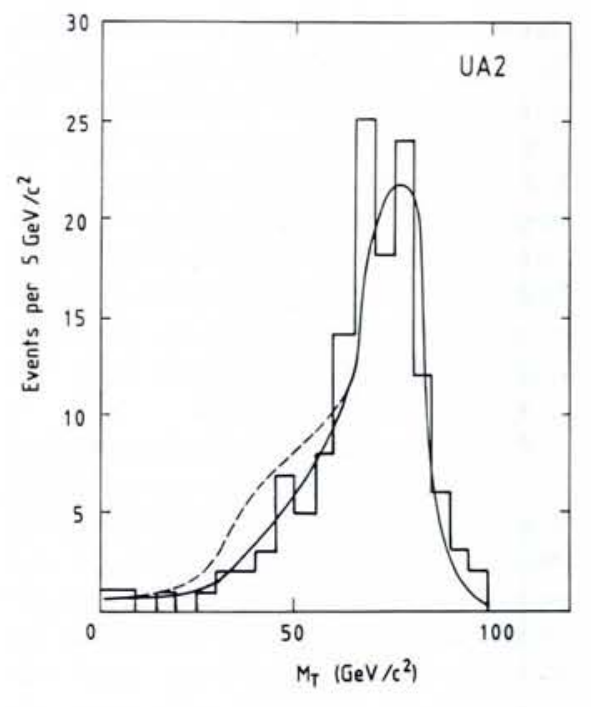

Fig. 5 - Transverse mass distribution for electron-neutrino pairs in the UA2 event sample. The full curve represents the best fit assuming no top signal. The difference between the dashed and full curve represents the contribution expected from the production and decay of a top quark with a mass of $65 \mathrm{GeV} / \mathrm{c}^{2}$.

\section{Results}

Large amounts of data have been recorded by the three experiments between September 1988 and June 1989. In all cases, the data were found to be statistically consistent with the expectation from conventional sources: they provided no evidence whatsoever for top quark production.

The most stringent bounds for the top mass value published so far have been obtained from studies of events containing an electron, a neutrino and one or more jets. In these events, a useful variable is the transverse mass of the electron-neutrino pair

$$
M_{\mathrm{T}}^{2}=2 p_{\mathrm{T}}^{\mathrm{e}} p_{\mathrm{T}}^{v}\left(1-\cos \Delta \phi_{\mathrm{ev}}\right)
$$

where $p_{T}^{\mathrm{e}}\left(p_{\mathrm{T}}^{v}\right)$ is the electron (neutrino) transverse momentum and $\Delta \phi_{e v}$ is the e- $v$ azimuthal separation. The $M_{T}$ distribution measured by UA2 (see Fig. 5 ) is in good agreement with expectations without top signal, and shows no evidence for the excess of events in the region $15<M_{\mathrm{T}}<50 \mathrm{GeV} / c^{2}$ expected from the production and decay of a heavy top (the top contribution for a mass of $65 \mathrm{GeV} / c^{2}$ is also shown in Fig. 5). From a statistical analysis of the distribution of Fig. 5, it is possible to exclude with $95 \%$ confidence a signal from a top quark with a mass value in the range $30<m_{\text {top }}<69 \mathrm{GeV} / c^{2}$.

The CDF experiment at the Fermilab $\overline{\mathrm{pp}}$ collider is sensitive to heavier top quarks than UA2, because of the much larger production cross-section associated with the higher $\sqrt{ } s$ value (see Fig. 3). 
This experiment has searched for the production and decay of the top quark into final states containing an electron, a neutrino and at least two jets. A fit to the electron-neutrino transverse mass distribution excludes with $95 \%$ confidence a top quark with a mass between 40 and $77 \mathrm{GeV} / \mathrm{c}^{2}$.

The CDF experiment has also searched for a top signal in events containing an isolated, opposite-sign e $\mu$ pair as expected from $t \bar{t}$ production with one t-quark decaying to $\mathrm{ev}_{\mathrm{e}} \mathrm{b}$ and the other to $\mu v_{\mu}$ b. Such a final state has the advantage of a very low background despite the small decay branching ratio (2/81 of all produced $t \bar{t}$ pairs). Only one event, with $p_{\mathrm{T}}^{\mathrm{e}}=31.7 \mathrm{GeV} / c$ and $p_{\mathrm{T}}^{\mu}=$ $42.5 \mathrm{GeV} / c$, is found to satisfy this requirement, in agreement with an estimated number of 1.2 background events. In comparison, a signal of 7.5 events is expected from t $\bar{t}$ production with a top quark mass of $70 \mathrm{GeV} / \mathrm{c}^{2}$. This result excludes top quarks in the mass range from 28 to $72 \mathrm{GeV} / c^{2}$ with $95 \%$ confidence.

More recently, the CDF collaboration has extended the analysis to final states containing an electron or muon, a neutrino, two jets and an additional low- $p_{\mathrm{T}}$ muon, which is expected to result mainly from the decay $\mathrm{b} \rightarrow \mathrm{c} \mu^{-} \bar{v}_{\mu}$ (or $\bar{b} \rightarrow \bar{c}$ $\mu^{+} v_{\mu}$ ). No event with this configuration has been observed so the lower bound on the $m_{\text {top }}$ has been extended to 89 $\mathrm{GeV} / c^{2}$ with $95 \%$ confidence.

\section{Prospects for Discovery}

Despite the fact that the top quark has not been found, its existence is needed to explain the charge asymmetry observed experimentally in the reaction $\mathrm{e}^{+} \mathrm{e}^{-} \rightarrow \mathrm{b} \overline{\mathrm{b}}$ [11]. This is because in the fermion doublets of Table 1 only the states of negative helicity (spin component antiparallel to the momentum) are present, as required by the observation of maximal parity violation in all weak processes mediated by $\mathrm{W}$ exchange (for this reason the fermion doublets of Table 1 are often called left-handed doublets; their antiparticles are arranged into right-handed doublets to describe the observed maximal violation of charge conjugation). Without a top quark, no charge asymmetry would be observed in the reaction $\mathrm{e}^{+} \mathrm{e}^{-} \rightarrow \mathrm{b} \bar{b}$, which is mediated by $\gamma$ and $Z$ exchange, because in this case both helicity components of the $b$ field would be weak iso-spin singlets and the $\mathrm{Zb} \overline{\mathrm{b}}$ coupling would conserve parity and charge conjugation. The fact that the top quark is so much heavier than any other known elementary fermion is not a problem be-

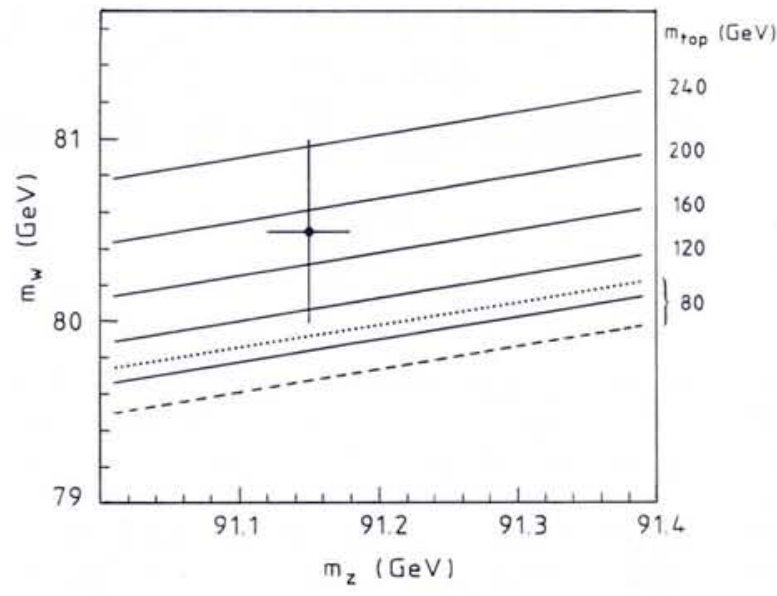

Fig. 6-Combined measurement of the $W$ and $Z$ masses from UA2 and the LEP experiments. The solid lines are Standard Model predictions for a given $m_{\text {top }}$ assuming a mass of $100 \mathrm{GeV} / \mathrm{c}^{2}$ for the Higgs, or Z, boson. The dotted (dashed) line is the prediction for $m_{\text {top }}=80 \mathrm{GeV} / \mathrm{c}^{2}$ and $a$ Higgs boson of 10 (1000) $\mathrm{GeV} / \mathrm{c}^{2}$. The measurement supports a top quark which is heavier than the Higgs boson.

cause the Standard Model cannot predict the values of the fermion masses.

The CDF result, $m_{\text {top }}>89 \mathrm{GeV} / c^{2}$ at the $95 \%$ confidence level, excludes the possibility that the top quark will be discovered at LEP in its present mode of operation $(\sqrt{ } s \leq 100 \mathrm{GeV})$, and leaves only a narrow window for the second phase of LEP operation at $\sqrt{ } s=200$ $\mathrm{GeV}$ in 1994. Over the next few years, the best chances for discovery are at the Fermilab $\bar{p} p$ Collider, which is expected to run again in the middle of 1991. Because of various improvements to the $\bar{p}$ source and to the collider itself, in a one-year long run the CDF experiment is expected to collect a data sample five times larger than the present one. In addition, a second major detector, D-ZERO, will begin data-taking. With a five-fold increase of the data sample, the search for a top signal becomes sensitive to $m_{\text {top }}$ values up to $\cong 120$ $\mathrm{GeV} / c^{2}$.

For top quarks heavier than the $\mathrm{W}$ boson, the dominant decay mode becomes $\mathrm{t} \rightarrow \mathrm{bW}^{+}\left(\mathrm{t} \rightarrow \overline{\mathrm{b}} \mathrm{W}^{-}\right)$. As a consequence, $t \bar{t}$ production results in final states which contain a $\mathrm{W}^{+} \mathrm{W}^{-}$pair. In this case, the search for a top signal in events containing an electron (or muon), a neutrino and one or more jets is made difficult by the fact that the ev (or $\mu v$ ) transverse mass distribution has the same shape as that expected from the much more abundant direct production of $\mathrm{W}$ bosons. The only way to search for top is to study $\mathrm{e}^{ \pm} \mu^{\mp}$ pairs or configurations containing an electron or muon, a neutrino, one or more jets and an additional low $p_{\mathrm{T}}$ muon.

Indirect evidence for a heavy top quark has also been obtained recently by precision measurements of the $\mathrm{W}$ and $Z$ mass values, which depend slightly on the top quark mass because of higher-order corrections arising from virtual loops in the boson propagators. This can be seen in Fig. 6 where the dependence of $m_{w}$ and $m_{z}$ on $m_{\text {top }}$ is shown as a series of lines in the $\left(m_{w}\right.$ $m_{z}$ ) plane. Also shown is a recent UA2 result on $m_{w}$, in combination with the precise value of $m_{z}$ measured at LEP. This data point is in good agreement with the Standard Model predictions and gives further support to a top quark which is heavier than the $\mathrm{W}$ boson.

We note finally that no additional fermion doublets beyond those shown in Table 1 are likely to exist. One of the first LEP results, probably the most important so far, has been the demonstration that the total number of neutrino types is $3.04 \pm 0.12$ [12] (this major discovery resulted from studies of the $Z$ line shape, because the decay $Z \rightarrow v \bar{v}$ with $m_{\mathrm{v}}<<m_{\mathrm{z}} / 2$ contributes $\approx 167 \mathrm{MeV}$ to the total width of the $Z$ boson for each neutrino type). We can conclude, therefore, that the top quark will be the last fundamental fermion to be discovered, unless the mass of the additional neutrinos is of the order of $40 \mathrm{GeV} / c^{2}$ or larger.

\section{REFERENCES}

[1] Gell-Mann M., Phys. Lett. B8 (1964) 214; Zweig G., CERN TH-412 (1964).

[2] Glashow S.L., lliopoulos J. and Maiani L., Phys. Rev. D2 (1970) 1285.

[3] See: Chinowsky W., Ann. Rev. Nucl. Part. Sci. 27 (1977) 393; Goldhaber G. and Wiss J.E., Ann. Rev. Nucl. Part. Sci. 30 (1980) 337.

[4] Herb S.W. et al., Phys. Rev. Lett. 39 (1977) 252

[5] See: Thorndike E.H., Ann. Rev. Nucl. Part. Sci. 30 (1980) 299.

[6] Bouchiat C., lliopoulos J. and Meyer Ph., Phys. Lett. B38 (1972) 519.

[7] Kamae T., Proc. XXIV Int. Conference on High Energy Physics, Munich, 1988 (Springer-Verlag, 1989) p. 156.

[9] Akrawy M.Z. et al., Phys. Lett. B236 (1990) 364; Decamp D. et al., CERN-EP/ 89-165 (1989).

[10] Altarelli G. et al., Nucl. Phys. B308 (1988) 724.

[11] Ash W.W. et al., Phys. Rev. Lett. 58 (1987) 1080; Elsen E. et al., DESY 89-127 (1989).

[12] See: Rolandi L., CERN-EP/90-64 (1990), 